\title{
Is Atypical Metastasis Obstructive to Sentinel Node Navigation Surgery in Early Gastric Cancer Patients?
}

\author{
Hiroya Takeuchi, MD, PhD, FACS and Yuko Kitagawa, MD, PhD, FACS \\ Department of Surgery, Keio University School of Medicine, Tokyo, Japan
}

Sentinel node navigation surgery (SNNS) is defined as novel, minimally invasive surgery which can avoid unnecessary lymph node dissection based on sentinel node (SN) biopsy and diagnosis for nodal metastasis targeting SN. In the past 10 years, several studies supporting the validity of the SN concept for early-stage gastric cancer have been reported. Single-institutional results of $\mathrm{SN}$ mapping for early gastric cancer are being increasingly considered acceptable in terms of detection rate and accuracy of predicting regional lymph node status. ${ }^{1-3}$

We recently conducted a multicenter, prospective trial of SN mapping for early gastric cancer using a dual-tracer method with a radioactive colloid and blue dye. ${ }^{4}$ In the trial, SN mapping was performed for 397 patients who had a single tumor (cT1N0M0 or cT2N0M0) with a primary lesion having a diameter of less than $4 \mathrm{~cm}$ and not undergone any previous treatments. As a result, the SN detection rate was $98 \%$, and the accuracy of nodal evaluation for metastasis was $99 \%$. The results of that clinical trial are expected to provide us with a perspective on the future of SNNS for early gastric cancer.

A recent article by Lee et al. ${ }^{5}$ reported on the pattern of lymphatic drainage in gastric cancer patients. They retrospectively evaluated the distribution of metastatic lymph nodes according to the longitudinal and circumferential location of tumors in $462 \mathrm{pT} 1$ or pT2 gastric cancer patients with metastasis to 1-3 lymph nodes. A considerable number of atypical metastases (skip or transversal metastasis) were observed, regardless of tumor location. In particular, tumors in the lower third of the stomach and/or

(C) Society of Surgical Oncology 2013

First Received: 6 November 2013;

Published Online: 4 December 2013

H. Takeuchi, MD, PhD, FACS

e-mail: htakeuchi@a6.keio.jp lesser curvature side had a significant propensity to exhibit atypical metastasis. They concluded that when applying SNNS in gastric cancer patients, care should be taken with tumors at these locations to prevent false-negative results when applying SNNS in gastric cancer patients.

Although this finding should be interpreted with caution as Lee et al. ${ }^{5}$ did not attempt SN mapping in their study, they have provided a valuable reference for the clinical application of SNNS in early gastric cancer patients. In fact, the pattern of lymph node metastasis, including atypical metastasis, in their study was remarkably similar to the distribution of $\mathrm{SN}$ in our previous multicenter trial. ${ }^{4}$ For instance, in our trial, the tumors in the lower third of the stomach had SNs at \#8a (in the second compartment) with relatively high probability. This result is in agreement with that obtained by Lee et al. ${ }^{4,5}$ Thus, the similarity of these two results suggests that most of atypical metastasis in their study may not represent false-negative SNs but 'typical and true' metastasis to SNs via aberrant lymphatic vessels from the primary tumor. We believe that most false-negative SNs may be owing to technical issues of SN biopsy rather than atypical metastasis.

Meanwhile, in our previous trial, SNs were located out of the area of D2 lymph node dissection in $1 \%$ of cases with primary tumors in the upper third, $3 \%$ in the middle third, and $6 \%$ in the lower third of the stomach, respectively. ${ }^{4}$ Therefore, caution needs to be taken regarding the application of D2 lymph node dissection even for early gastric cancer.

In future studies, appropriate indications for functionpreserving gastrectomy, including proximal gastrectomy, segmental gastrectomy, pylorus-preserving gastrectomy, and partial resection for cT1N0 gastric cancer, should be individually determined on the basis of SN mapping. ${ }^{3}$ Various types of laparoscopic function-preserving surgeries can be performed in gastric cancer patients with no metastatic SNs. Previous reports on SN biopsy have 
demonstrated that sentinel lymphatic basins contain truly positive nodes, even when SN biopsy produces a falsenegative result. ${ }^{3,4}$ Sentinel lymphatic basin dissection can provide an acceptable safety net for the clinical application of laparoscopic SN sampling for gastric cancer. ${ }^{3}$ The pattern of sentinel lymphatic basin could be approximately estimated by primary tumor location according to the accumulation of the results of SN mapping in cT1N0 gastric cancer. ${ }^{6}$ However, at the same time, the fact that distribution of SN shows an unpredictable pattern in some patients, as demonstrated by Lee et al., ${ }^{5}$ should also be taken into account. We believe that intraoperative SN mapping and biopsy are indispensable for verifying that all SNs are negative for cancer metastasis, and for deciding the actual extent of the sentinel lymphatic basin in case we attempt the laparoscopic function-preserving gastrectomy.

For early-stage gastric cancer such as cT1NOM0, for which a better prognosis can be achieved through conventional surgical approaches, the establishment of individualized, minimally invasive treatments that may preserve the patients' quality of life should be the next surgical challenge. As a diagnostic tool, SN mapping should be extremely accurate for the identification of clinically unpredictable lymph node metastasis. Thus, further studies are needed to minimize false-negative results of SN biopsy due to technical errors. Nonetheless, we desire that SN mapping will have proven to be a promising strategy for minimally invasive, individualized management in patients with early-stage gastric cancer.

\section{REFERENCES}

1. Kitagawa Y, Fujii H, Mukai M, et al. The role of the sentinel lymph node in gastrointestinal cancer. Surg Clin North Am. 2000;80:1799-809.

2. Kitagawa Y, Fujii H, Mukai M, et al. Radio-guided sentinel node detection for gastric cancer. Br J Surg. 2002;89:604-8.

3. Takeuchi H, Kitagawa Y. New sentinel node mapping technologies for early gastric cancer. Ann Surg Oncol. 2013;20:522-32.

4. Kitagawa Y, Takeuchi H, Takagi Y, et al. Sentinel node mapping for gastric cancer: a prospective multicenter trial in Japan. J Clin Oncol. 2013;31:3704-10.

5. Lee JH, Lee HJ, Kong SH, et al. Analysis of the lymphatic stream to predict sentinel nodes in gastric cancer patients. Ann Surg Oncol. In press.

6. Kinami S, Fujimura T, Ojima E, et al. PTD classification: proposal for a new classification of gastric cancer location based on physiological lymphatic flow. Int J Clin Oncol. 2008;13:320-329. 